\title{
Wild Game: Fresh Meat Processing ${ }^{1}$
}

\author{
Michael Fioretto, Chad Carr, Jason M. Scheffler, Jennifer G. Bearden, and Halie Corbitt ${ }^{2}$
}

\section{Processing Food Safety}

The key to minimizing food safety risk and maximizing the shelf life of fresh food is to keep it clean and cold. Keeping it clean will minimize the initial microbial load on the food. Chilling perishable foods quickly after harvest and keeping them cold will minimize the time for exponential microbial growth. Bacteria grow between $40^{\circ} \mathrm{F}$ and $140^{\circ} \mathrm{F}$; this range is often referred to as the "danger zone." While you may face some limitations in the ability to chill the meat, it is best to get the meat to refrigeration temperature $\left(35^{\circ} \mathrm{F}-40^{\circ} \mathrm{F}\right)$ as quickly as possible. While chilling the meat will not kill the bacteria, keeping meat below $40^{\circ} \mathrm{F}$ will limit bacteria growth and significantly decrease the risk of foodborne illness.

When preparing to process fresh meat, you need to avoid introducing bacteria to the meat. The environment should be as clean as possible. Wash your hands very well before handling meat. Additionally, plastic gloves should be worn while processing meat. You should sanitize all surfaces and any equipment you plan to use, such as knives, saws, or grinders.

\section{Deciding What Products You Want}

The steps you take in processing your wild game meat will change depending on the final product(s) that you desire. There are two main routes to take: making some whole muscle cuts (steaks and roasts) or turning it all into ground meat. Note that when cutting for whole muscle cuts, there will still be a portion of the carcass that will be ground, so you will have ground meat regardless of your decision. When making this decision, you should consider the dining experience you would like, the processing requirements for each process, and the shelf life of the product.

Whole muscle cuts can be cooked to a lower end temperature, such as medium-rare. These cuts can be cooked to lower temperatures because the inside of the muscle remains sterile (unpenetrated). Examples of whole muscle cuts are roast, steaks, chops, backstrap, tenderloins, etc. If one plans to have whole muscle cuts, the carcass should be aged before processing into cuts to improve tenderness. If the carcass is not aged before processing, the meat will undergo the rigor process off the bone. This will result in a condition called "rigor shortening," where the muscle fibers will overly contract and shorten, and the meat will be denser and tougher. A more tender product will result if the carcass is aged, because rigor will occur on the bone, and the meat will not over-shorten. Furthermore, the aging process allows the muscle proteins to degrade somewhat, which further improves tenderness. Additionally, whole muscle cuts will likely need to be tenderized before cooking, because game meat is very tough. Another option is to make cube steaks out of any whole muscle steaks you plan on keeping. The cubing process is a simple process that mechanically tenderizes the meat, but this requires a meat cuber. Frequently, the limiting factor for most in making cube steaks is having this piece of meat processing equipment. Note that once you cube the meat, a higher

1. This document is FCS3365, one of a series of the Department of Family, Youth and Community Sciences, UF/IFAS Extension. Original publication date January 2022. Visit the EDIS website at https://edis.ifas.ufl.edu for the currently supported version of this publication.

2. Michael Fioretto, student, Department of Animal Sciences; Chad Carr, assistant professor, Department of Animal Sciences; Jason M. Scheffler, research assistant professor, Department of Animal Sciences; Jennifer G. Bearden, agriculture Extension agent II, UF/IFAS Extension Okaloosa County; and Halie Corbitt, family and consumer sciences Extension agent I, UF/IFAS Extension Columbia County; UF/IFAS Extension, Gainesville, FL 32611.

The Institute of Food and Agricultural Sciences (IFAS) is an Equal Opportunity Institution authorized to provide research, educational information and other services

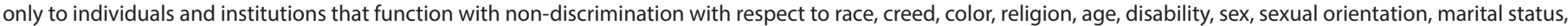

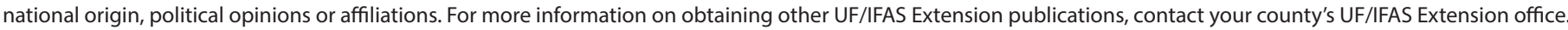
U.S. Department of Agriculture, UF/IFAS Extension Service, University of Florida, IFAS, Florida A \& M University Cooperative Extension Program, and Boards of County Commissioners Cooperating. Andra Johnson, dean for UF/IFAS Extension. 
cooking temperature will be required. Finally, whole muscle cuts will have a longer shelf life than ground meat, both refrigerated and frozen.

Ground meat will need to be cooked to a well-done temperature of $160^{\circ} \mathrm{F}$. It cannot be cooked to a lower temperature like whole muscle cuts, because the grinding introduces potential pathogens into the once sterile interior of the muscle. There is no need to worry about tenderness with ground meat because the grinding process breaks apart the muscle fibers; therefore, aging the carcass before processing is not necessary. However, you can still age the carcass if it is desired. Note that ground meat will have a shorter shelf life than whole muscle cuts.

\section{Aging}

When aging a carcass, the meat should be held at refrigeration temperatures $\left(35^{\circ} \mathrm{F}-40^{\circ} \mathrm{F}\right)$. If the meat is held at higher temperatures, there can be problems with food safety because bacteria will multiply. Conversely, if meat is frozen, the textural changes that need to occur during the aging process will not occur. If meat is frozen before it can undergo rigor, the meat will experience a condition called "thaw rigor." In thaw rigor, the muscle will undergo the rigor process while thawing. This will result in the muscle over-contracting and over-shortening, making for very tough meat.

During aging, meat can either be hung as the whole carcass or in quarters. Processing the carcass into any smaller cuts will result in trouble with rigor shortening, explained in the "Deciding What Products You Want" section. If the carcass is going to be aged whole, it is recommended, but not required, to age the carcass with the hide on. Aging with the hide on will minimize the loss of water from the carcass, increasing yield. Additionally, the hide will help to keep the carcass clean. If the hide is removed, it is recommended to cover the carcass with cheesecloth or a similar cover to help keep it clean.

Deer can be aged up to seven days. The desired changes to the muscle will have occurred in this time frame. If the deer is left to age more than seven days, there are potential problems with bacterial growth and potential foodborne illness. If the hunting wound resulted in notable carcass contamination, then the aging time would need to be reduced to account for additional microbial presence from the contamination.

\section{Carcass Cutting}

Once aging is complete, the carcass can be cut and portioned into various desired cuts. Before and during cutting and portioning, some important parts of the carcass should be removed. It is recommended to remove the musk or scent glands when taking the hide off the animal; however, it is not necessary. When removing these glands, take care not to puncture them. During cutting, tendons and other heavy connective tissue need to be removed because they are inedible. There is heavy connective tissue in many parts of the entire carcass, but there are a few specific places to look out for it. These include the backstrap connective tissue, which runs along the length of the backbone, and the tendons located in the shanks. Furthermore, it is recommended to remove all bones from any whole muscle cuts you plan on keeping. Having boneless cuts is a safety precaution against eating product from animals not yet showing signs of early-stage chronic wasting disease (CWD). However, removing meat from the bone will not make meat safe if animals are already exhibiting signs of CWD; these animals should be avoided. CWD has not yet been reported in Florida. Additionally, any discolored meat, likely to be found near the hunting wounds, should be removed and discarded. Finally, it is recommended, but not required, to trim fat from the meat during cutting. Fat is the main driver of flavor in the meat from any animal species. While game animals tend to be fairly lean, the fat on a carcass carries a widely undesirable flavor, often described as gamey. The removal of fat is essential when cutting and portioning boars due to boar taint. Boar taint is a result of male steroid hormones affecting the quality and flavor of the meat. However, these hormones are present in high amounts in fat; thus, removing the fat will greatly reduce the impact of boar taint on the meat eating quality.

Cutting and portioning should be performed using the following steps. The detailed process is adapted from "You and Your Wild Game" (Field \& Raab, 1983). These steps are written for processing from a whole carcass; however, these same steps can still be followed to cut and portion quarters as well.

1. At the base of the neck, remove the neck from the shoulder, using a bone saw to get through the vertebrae. Debone the neck and grind the meat.

2. Separate the shoulder from the rib between the fifth and sixth ribs, using a bone saw to cut through the vertebrae, blade bone, and sternum. 
3. The brisket should be removed from the shoulder just above the elbow joint.

4. From the shoulder, cut arm and blade roasts or steaks. Portions from the shoulder not suitable for roasts or steaks should be turned into ground meat or stew meat.

5. The foreshank and brisket should be deboned and used for either ground meat or stew meat.

6. The leg should be separated from the loin, where one lumbar vertebrae should remain on the leg. A bone saw should be used to cut through the vertebrae.

7. From the leg, sirloin steaks and leg roasts can be cut. The hind shank and other trimmings not suitable for roasts and steaks can be deboned for ground or stew meat.

8. Separate the rib and breast from the flank and loin between the twelfth and thirteenth rib, using a bone saw to cut through the vertebrae.

9. Separate the breast from the rib with a cut parallel to the back. The cut should be $1 / 2$ inch ventral (towards belly) from the blade bone.

10. The rib can be left whole as a roast or cut into steaks.

11. Spare ribs can be cut from the breast or can be entirely turned into ground or stew meat.

12. Separate the flank from the loin with a cut alongside the tenderloin, parallel to the back.

13. The tenderloin can be removed from the rest of the loin and turned into roasts or steaks, or the tenderloin can be left on the loin and be a part of loin cuts.

14. The loin can be left whole as a roast or cut into steaks.

15. The flank should either be ground up for ground meat or cubed for stew meat.

\section{Grinding Meat}

1. Mix $10 \%-30 \%$ by weight beef fat or pork fat with game meat to be ground. This is to make the final product juicier and give it a more desirable flavor.

2. It is recommended to grind meat while it is at refrigerated temperatures, rather than at room temperature. This will result in a better and more consistent grind, as well as improved food safety.
3. Using a grinder and coarse grinder plate, make an initial coarse grind of the fat and lean mixture.

4. Mix the coarse ground product to ensure even fat distribution.

5. Using a grinder and a fine grinder plate, make a final grind of the product.

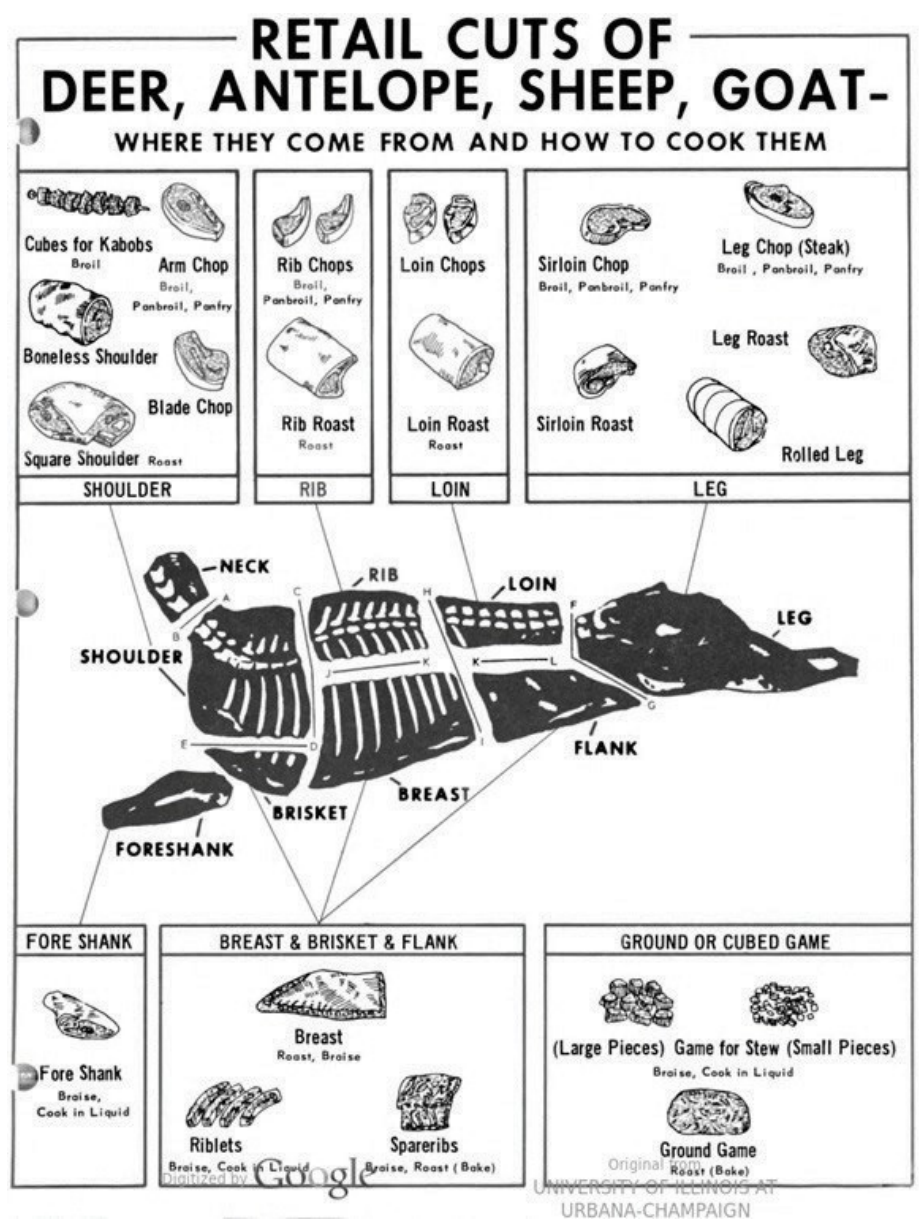

Figure 1. Retail cuts of deer, antelope, sheep, and goat.

Credits: University of Illinois at Urbana-Champaign. Used with permission.

\section{Shelf Life}

The shelf life of game products depends on a couple of different factors. One factor that we have already mentioned is whether or not the product is whole muscle or ground. Ground meat tends to have a shelf life approximately half that of whole muscle cuts. Additionally, the storage temperature has a significant impact on shelf life, with frozen product having an exponentially longer shelf life than refrigerated product. If product is going to be refrigerated, then the packaging it is stored in will affect the shelf life. Vacuum (anaerobic) packaging will keep meat fresh longer, whereas non-vacuum (aerobic) packaging will result in a shorter shelf life. Finally, a cooked meat product will have a 
shorter shelf life than an uncooked meat product. A summary of shelf life based on these different factors is below.

\section{Refrigerated shelf-life $\left(35^{\circ} \mathrm{F}-40^{\circ} \mathrm{F}\right)$}

- Non-vacuum/Aerobic packaging

- Whole muscle cuts-Up to a week

- Ground or cooked-Less than 5 days

- Vacuum/Anaerobic packaging

- Fresh meat-Up to a month

Frozen shelf life (below $0^{\circ} \mathrm{F}$ )

- Well-packaged whole muscle cuts-1 year

- Ground-Up to 6 months

- Cooked-2-3 months

\section{Freezing}

Unless the game meat is to be eaten within a few days after processing, it will need to be frozen. You can freeze meat with a few different packaging materials. Plastic vacuum packaging can be used and is very effective; however, a vacuum sealer is necessary. You can also package meat for freezing by wrapping the product with butcher paper (waxed paper), freezer wrap, or aluminum foil. When packaging with one of these materials, wrap the product as tightly as possible to minimize the air within the package. This will help to maintain the shelf life, even though the product is frozen.
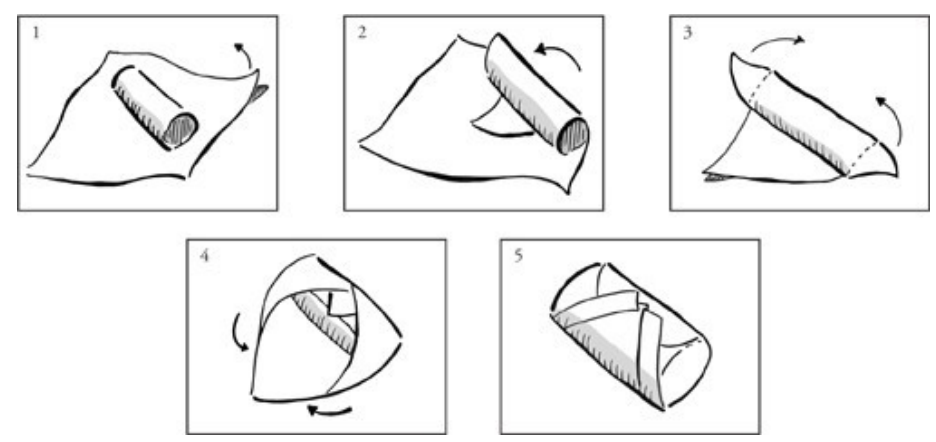

Figure 2. Diagram of steps to follow when wrapping meat for freezing. Credits: University of Georgia Extension. Used with permission.

When portioning and packaging meat to be frozen, meat should be packed into meal-sized portions. Generally, this will be about a pound of meat per individual package. Packing meat into meal-sized portions will prevent too much meat from being thawed at one time. Additionally, a permanent marker should be used to label each package of meat before freezing. The package should be labeled with the cut and the freeze date.
To maintain the highest food quality and safety, freezing needs to be performed as quickly as possible. A blast freezer that can rapidly freeze meat is ideal; slower freezing will result in a lower-quality product. A rapid freeze will more effectively impede rancidity and bacterial growth and cause the formation of smaller ice crystals. Freezing can be accelerated by spacing meat out in the freezer. If there is space between cuts of meat in the freezer, cool air can pass between each cut to help the products freeze faster. Depending on the type of freezer or the amount of freezer space you have, you may be limited in your ability to freeze meat very quickly. However, as long as the meat is frozen within a reasonable time frame, the product will be perfectly safe. Additionally, frozen meat should be stored at or below $0^{\circ} \mathrm{F}$ to maintain the best quality.

\section{Thawing}

Proper thawing of meat can be just as important to maintaining food safety as proper freezing is. Meat can be properly thawed in a refrigerator, cold-water bath, or microwave. Meat should NOT be thawed on the counter. If meat is thawed on the counter, there is a potential risk of cross-contamination. Additionally, if the meat reaches room temperature, accelerated microbial growth can occur, which can affect food safety.

Thawing meat in the refrigerator takes the longest but is the easiest and safest method. If you forgot you were thawing out a cut, then it would still be held at refrigerated temperatures. When thawing in a refrigerator, the meat should be thawed in the same package in which it was frozen. It will take approximately 3-7 hours per pound of meat to thaw ground product. A 1-inch thick steak will take approximately $12-14$ hours to thaw.

Thawing meat in a cold-water bath takes much more attention but is a quicker alternative to thawing in a refrigerator. The water from the thaw bath needs to be replaced every 30 minutes so that the meat continues to thaw. The bath must contain COLD water so that the meat does not reach too high of a temperature. To thaw in a cold-water bath, the meat must have been frozen in an airtight plastic package, or the entire package would need to be placed into a leak-proof plastic bag. It will take approximately 45 minutes per pound of meat to thaw in a cold-water bath.

Thawing in a microwave should generally be done as a last resort, but it is very quick. Due to differences among microwaves and their settings, it is best to follow the directions from the microwave manual to thaw meat using this method. However, even large roasts can generally be 
thawed within 20 minutes. When thawing in a microwave, plan to cook the meat immediately after thawing is complete, because some parts of the product may have gotten warm and even started cooking during thawing.

\section{References}

CDC. (2021). Chronic Wasting Disease. Retrieved from

https://www.cdc.gov/prions/cwd/occurrence.html

Cutter, C. N. (2020). Proper Processing of Wild Game and Fish. University Park: Penn State Extension.

Cutter, C. N. (2011). Proper Care and Handling of Venison from Field to Table. University Park: Penn State Extension.

Field, R. A., \& Raab, C. A. (2010). You and Your Wild Game. Laramie, WY: University of Wyoming Agricultural Extension Service. 